\title{
A Temporal Proper Orthogonal Decomposition (TPOD) Method for Closed-Loop Flow Control
}

\author{
Stanislav Gordeyev ${ }^{1}$ and Flint O. Thomas ${ }^{2}$ \\ University of Notre Dame, Notre Dame, IN 46556, USA
}

\begin{abstract}
Motivated by closed-loop flow control applications, a new formulation of the proper orthogonal decomposition (POD) is presented which is capable of characterizing not only the controlled and natural states of a given flow but also the transient behavior between these states. This approach, which is termed temporal POD (TPOD) extracts the optimum frameof-reference and the temporal information regarding the dynamics of the system in the presence of the flow control. The TPOD concept is developed in this paper and demonstrated experimentally in an application involving a circular cylinder in cross flow at $\mathbf{R e}_{\mathrm{D}}=\mathbf{5 , 0 0 0}$ with active plasma flow control. The resulting model is shown to properly capture the correct dynamics of the first TPOD mode, including the natural, forced and transient regimes.
\end{abstract}

\section{Motivation and Objectives}

$\mathrm{C}$ ONVENTIONAL proper orthogonal decomposition (POD)-based modeling extracts an optimal complete set of dominant (in the energy sense) spatial modes, with a minimum number of modes to represent a particular flow state $^{1-3}$. These modes are usually projected onto the Navier-Stokes (N-S) equations to obtain a low-dimensional system of ODE equations to describe the temporal evolution of the modes ${ }^{4,5}$.

This approach is shown to give satisfactory results, if the flow does not deviate much from a given state, such as the natural evolution of a shear layer or jet, for instance. But when the flow is actively manipulated using some form of flow control, the set of POD modes extracted from the natural flow state typically does a very poor job describing the forced or controlled flow state. From a topological point of view, this can be explained as shown in Figure 1: for the natural state the dynamical system (the flow) occupies a particular finite region in phase space. POD extracts a set of eigenvectors (modes) and provides a low-dimensional frame of reference to describe the system evolution within this region. When the flow control is activated, the system is forced to travel and to occupy a different finite region in the phase space. So, the set of POD modes derived to describe the system evolution in one region with as few eigenvectors as possible becomes quite non-optimal for another region, and, although the set of POD modes is still complete, it results in dramatic increase in the number of POD modes needed to provide a proper frame of reference to describe the forced system.

To address this issue, several modifications of the basic POD approach have been proposed to solve this problem. For periodic flows, the double POD or DPOD approach ${ }^{6,7}$ provides a set of POD modes calculated within each period, and performs a second POD-optimization among POD

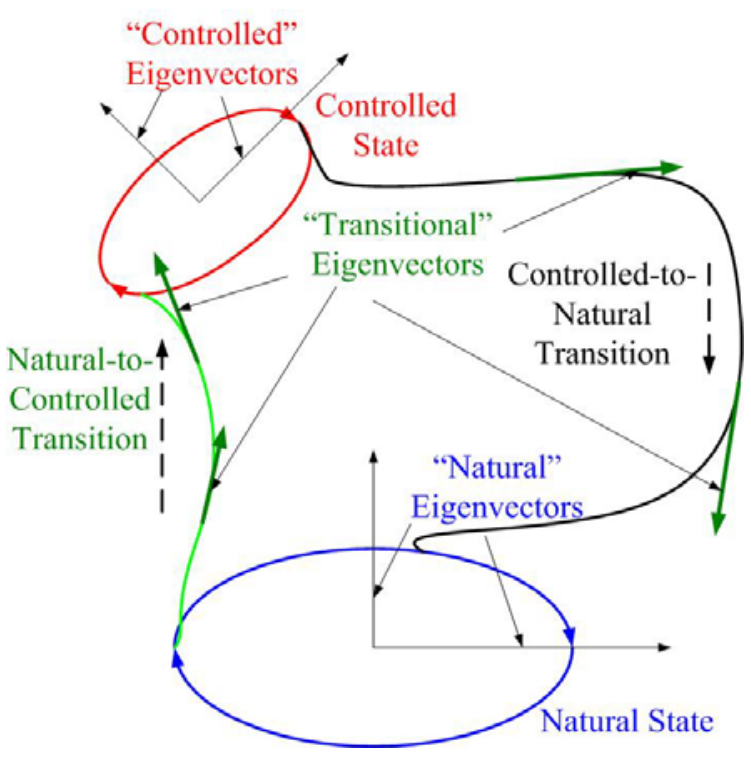

Figure 1. Topology of the dynamical system in phase space.

\footnotetext{
${ }^{1}$ Research Assistant Professor, AME Department, 120 Hessert Laboratories, AIAA Member.

2 Professor, AME Department, 108 Hessert Laboratories, AIAA Associate Fellow.
} 
modes between periods, thus providing the eigenvector basis to optimally describe the natural, the transient and the forced states. However, the method may not be readily extended to aperiodic flows. Split POD ${ }^{8}$ provides the extended POD basis, which is optimal for both the natural and the forced states, but may not be optimal for the transient state, thus potentially losing information of how the system goes from the natural to the forced state and back. Balanced POD ${ }^{9}$ was demonstrated to capture proper dynamical modes from the flow, but can be used only in computational studies, since it requires the solution of the adjoint problem, though recently a technique called Eigensystem Realization Algorithm ${ }^{10}$ was shown some promise to calculate Balanced POD modes directly from the experiments ${ }^{11}$.

All of the mentioned techniques extract an optimal set of spatial eigenmodes to describe the system evolution with the presence of the flow control. However, to extract the temporal evolution or trajectories of these modes, they rely on either the Galerkin projection into the N-S equations or an empirical set of temporal equations. The former approach usually suffers from the closure problem, while the second one requires significant amount of empirical knowledge about the system dynamics.

We propose an alternative to the approaches outlined above; a new time-dependent version of the POD procedure is described in the following section. For the sake of simplicity we describe a 2-dimensional version of it, with a straightforward extension to a 3-dimensional version. To demonstrate the effectiveness of the proposed TPOD technique, the flow behind the circular cylinder at a moderate Reynolds number was chosen. This flow has well-understood vortical-street dynamics ${ }^{11}$ and had been extensively studied using different low-dimensional techniques, see [6] and references therein. In Section III the experimental set-up, the principle of operation of the plasma actuator as a flow control device and the velocity-field extraction procedure are described. In Section IV we apply the TPOD technique to the experimental PIV snapshots of the instantaneous velocity field downstream of the cylinder with and without the flow control to extract the dominant TPOD mode and to develop the simple non-linear model to correctly predict its dynamical evolution with the presence of the flow control. Preliminary conclusions and future work are discussed in Section V.

\section{Time-Dependent POD Approach (TPOD)}

The system at any moment, $t$, is described by an instantaneous field, $u(x, y, t)_{\alpha}$, where $u$ is the velocity field and $\alpha$ denotes a particular realization. Flow control is activated at time $t=t_{0}$, so the system is in the natural state $u^{N}(x, y, t)_{\alpha}$ for $t<t_{0}$ and it is in the forced or controlled state $u^{C}(x, y, t)_{\alpha}$ for $t-t_{0} \rightarrow \infty$.

The traditional low-dimensional POD approach ${ }^{1-3}$ consists of computing eigenvectors $\left\{\varphi_{i}(x, y)\right\}$ for any stationary state as solutions of the eigenvalue problem,

$$
R_{\beta \gamma}\left(x, y ; x^{\prime}, y^{\prime}\right) \varphi_{\gamma}\left(x^{\prime}, y^{\prime}\right)=\lambda \varphi_{\beta}(x, y),
$$

where, $R_{\beta \gamma}\left(x, y ; x^{\prime}, y^{\prime}\right)=\overline{u(x, y, t) u\left(x^{\prime}, y^{\prime}, t\right)}$ is a cross-correlation matrix, eigenvalue $\lambda_{i}$ gives the average energy of each mode 2 and the overbar denotes averaging in the time-domain. The system is then typically approximated by a (relatively) small number of modes. After projecting the truncated set onto the N-S equations, the system of ODEs can be derived as,

$$
\frac{d a_{i}(t)}{d t}=A a_{i}(t)+Q(a, a)+N(a),
$$

where $A, Q$ and $N$ are constant matrices, responsible for linear mode evolution (convection, production and dissipation), quadratic interaction between modes (energy transfer) and non-linear stabilizing terms, respectively.

Both the natural and controlled states are stationary states, so they can be described by separate sets of POD modes, $\left\{\varphi_{i}^{N}(x, y)\right\}$ and $\left\{\varphi_{i}^{C}(x, y)\right\}$, respectively. These sets describe the system in both states, but the very important information about how the system gets from the natural to the controlled state is completely lost during time-averaging. So in order to extract the trajectory information, another averaging operator is needed.

Note that by activating the flow control at time $t=t_{0}$, the system becomes non-stationary and therefore it can be presented as $u\left(x, y, \tau=t-t_{0} ; t_{0}\right)$. Here $\tau$ is the time delay from the initiation of actuation, which describes the system trajectory in time and $t_{0}$ defines where this trajectory starts, that is provides the initial condition of the trajectory. Topologically it can be explained as follows: at any given fixed $t_{0}$ the system leaves the natural state and goes toward the control state along a particular trajectory from a set of trajectories (since the system is stochastic), see Figure 2. We propose to define this set of trajectories using POD approach as follows: 
1. For a fixed $t_{0}$, an ensemble of system trajectories is collected, $\left\{u\left(x, y, \tau ; t_{0}\right.\right.$ - fixed $\left.)\right\}$.

2. For a given time delay, $\tau$, POD modes are calculated by ensemble-averaging over many realizations, so time-dependent POD modes or TPOD modes, $\left\{\tilde{\varphi}_{i}(x, y ; \tau)\right\}$, and corresponding mode energies, $\lambda_{i}(\tau)$, are functions of the time delay.

3. The system can be represented as a summation of the first few TPOD modes,

$$
u\left(x, y, \tau ; t_{0}-\text { fixed }\right) \approx \sum_{i=1}^{M} c_{i} \sqrt{\lambda_{i}(\tau)} \tilde{\varphi}_{i}(x, y, \tau)=\sum_{i=1}^{M} c_{i} \varphi_{i}(x, y, \tau) .
$$

Here coefficients $c$ are ensemble-averaged energyrelated weights of the corresponding TPOD modes.

The first TPOD mode represents the temporal evolution or a trajectory of the most energetic mode in the flow when the flow control is activated. Each TPOD mode is a function of space and the time-delay and it gives valuable information about how dominant flow structures evolve as a result of the flow control.

It is convenient to project each spatio-temporal TPOD mode, $\phi_{i}(x, y, \tau)$ into a set of spatial modes, $\psi_{i, j}(x, y)$ as

$$
\varphi_{i}(x, y, \tau)=\sum_{j} a_{i, j}(\tau) \psi_{i, j}(x, y),
$$

The set of finite number of $\Psi_{(i, j)}$-POD modes provides the most optimal set of eigenvectors to describe the natural, transient and control states. The temporal coefficients, $a_{i, j}(\tau)$, define trajectories in this eigenvector phase-space.

By construction, TPOD modes are function of $t_{0}$, so to describe the full system, a set of TPOD modes,

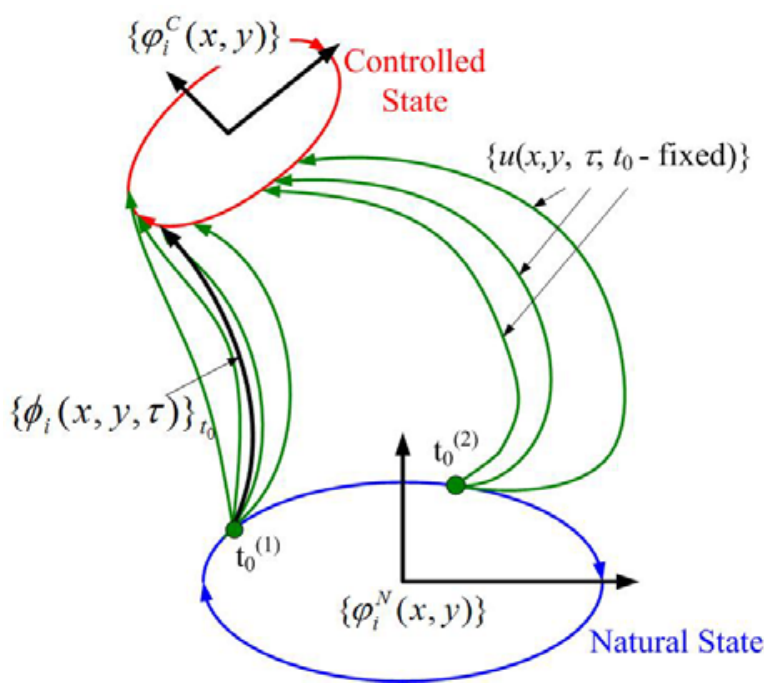

Figure 2. Topological definition of TPOD modes. $\left\{\phi_{i}\left(x, y ; \tau ; t_{0}\right)\right\}_{t_{0}}$ for each $t_{0}$ should be extracted from the experiment by means of conditional sampling. In the case, when the natural state is periodic and can be described as a closed orbit, TPOD-mode extraction is reduced to the conditional sampling as a function of the phase along the closed natural orbit.

It is worth noting that from Eqs. (3) and (4) it follows that $\Psi$-modes can be calculated directly from the original velocity field, $u$, by averaging all velocity fields in time and computing the correlation matrix, $R$, (1), but the temporal evolution of these modes will be lost in the time-averaging procedure.

The original system is governed by N-S equations and the Galerkin projection of the set of standard POD modes onto the N-S equations gives the system of ODE's to describe temporal evolution of coefficients for each standard POD mode. In general, this system has linear, quadratic and higher non-linear terms $(2)^{1-3}$. When POD modes are extracted experimentally, this approach involves the numerical calculation of the spatial derivatives, which typically suffers from experimental error. Also, the truncated system of equations requires some kind of closure to make the system stable.

Several approaches were developed to address these issues. Artificial neural network (ANN-ARX) ${ }^{6,7}$ was used to develop a stable system of equations to describe the wake evolution behind a cylinder flow in the presence of forcing. Other researchers look for the set of equations in a specified form, inspired by studying the dynamics of the system. ${ }^{13,14}$ A similar, but more general approach, used in [15] postulated the presence of linear, quadratic and cubic terms in (2) and found corresponding matrices and tensors using time-dependent POD coefficients extracted from the experiment via the least-square analysis.

Adapting the approach from [15], we also seek the temporal evolution of TPOD modes as a combination of the linear, quadratic and cubic terms,

$$
\frac{d \vec{a}(\tau)}{d \tau}=A \vec{a}(\tau)+Q \vec{a}(\tau) \vec{a}(\tau)+T \vec{a}(\tau) \vec{a}(\tau) \vec{a}(\tau)+B f,
$$

where $A, Q, T$ and $B$ are assumed to be constant matrices and tensors. The effect of the forcing "strength", $f$, is modeled by the linear term $B f$, similar to $[13,14,16]$. Since the temporal evolution of each TPOD mode is known from (4), the system (5) can be solved to find $A, Q, T$ and $B$ using the least-squared analysis, 


$$
\int_{\tau}\left[\frac{d \vec{a}(\tau)}{d \tau}-(A \vec{a}(\tau)+Q \vec{a}(\tau) \vec{a}(\tau)+T \vec{a}(\tau) \vec{a}(\tau) \vec{a}(\tau)+B f)\right]^{2} d \tau \rightarrow \min
$$

and now the original system is modeled by the set of ODE's (5).

\section{Experimental Set-up}

In order to experimentally demonstrate the TPOD approach, active control of the flow over a circular cylinder in cross-flow using dielectric barrier discharge (DBD) plasma actuators ${ }^{17}$ is used. Effective control of the cylinder in cross-flow using DBD plasma actuation has been demonstrated in previous studies. ${ }^{18,}{ }^{19}$ Plasma actuators were demonstrated to have a dramatic effect on the cylinder wake flow with the complete elimination of unsteady Karman shedding in the wake. Consequently, the flow has two very distinct states: the natural state with unsteady Karman shedding of large-scale vortices into the wake and the controlled state, where the separation region is greatly reduced in size and shedding is essentially completely suppressed. These two states are clearly illustrated in the sample flow visualization images shown in Figure 3. The flow visualization is obtained by seeding the flow with 1 micron diameter olive oil droplets which are illuminated by a pulsed Nd-Yag laser.
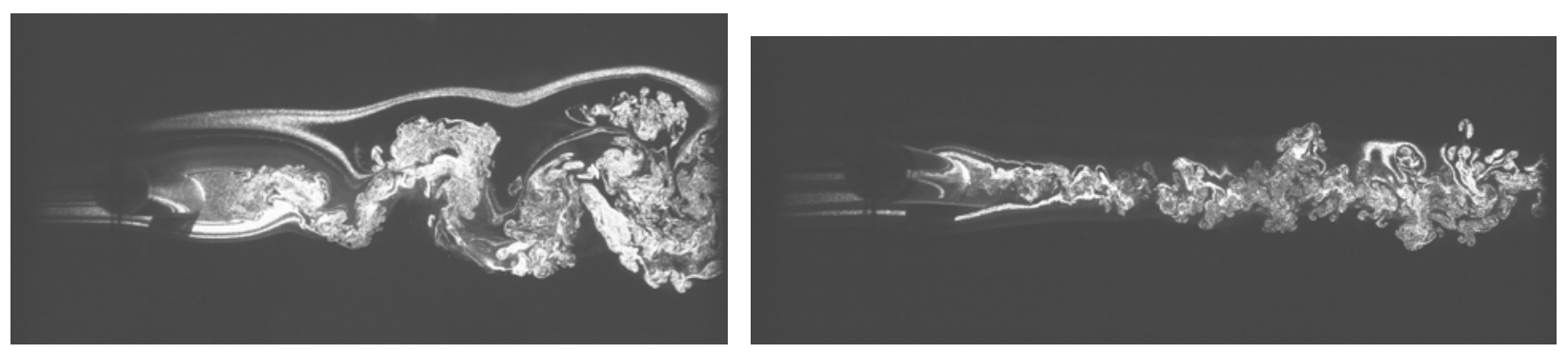

Figure 3. Flow visualization of the flow over the circular cylinder at $\operatorname{Re}_{D}=5,000$ : (left) the natural state, (plasma off), (right) the controlled state (plasma on).

The flow control experiments are performed in one of the low-turbulence, subsonic, in-draft wind tunnels located at the Hessert Laboratory for Aerospace Research at the University of Notre Dame. The wind tunnel has an inlet contraction ratio of 20:1. A series of 12 turbulence management screens at the front of the inlet give rise to tunnel freestream turbulence levels less than $0.1 \%(0.06 \%$ for frequencies above $10 \mathrm{~Hz})$. Experiments are performed in a test section of $0.610 \mathrm{~m}$ square cross-section and $1.82 \mathrm{~m}$ in length. One sidewall and the test section ceiling has optical access for non-intrusive laser flow field diagnostics (in this case Particle Image Velocimetry).

The cylinder model is shown schematically in Fig. 4. It consists of two coaxial fused quartz tubes: $13.5 \mathrm{~mm}$ I.D. $x$ 19mm O.D. x 533.4mm long and 6mm I.D. x 13mm O.D. x 533.4mm long. The dielectric constant of quartz is 3.7. The ends of the cylinder terminate in plastic endplates which elongate the model by $19.05 \mathrm{~mm}$. The wall of the outer tube serves as the dielectric barrier for the DBD plasma actuators. The exposed and insulated electrodes are common for both plasma actuators due to space limitations. These electrodes are made of Saint Gobain C661 $1.6 \mathrm{mil}(0.041 \mathrm{~mm})$-thick copper foil tape with acrylic adhesive. As indicated, the outer, exposed electrode (2) is mounted to the outer surface of the outer quartz tube with its plasma generating edges located at \pm 90 degrees with respect to the approach flow direction (left-to-right in Figure 4). The insulated electrode (3) is mounted to the outer surface of the inner quartz tube (4). Both inner and outer electrodes extend $444.5 \mathrm{~mm}$ in the spanwise direction. The inner and outer electrodes have a small overlap, which gives rise to a large local electric field. Plasma (5) forms near the edge of the exposed electrode and extends a distance along the cylinder's dielectric surface as depicted in Fig. 4. As indicated in the figure, the actuators

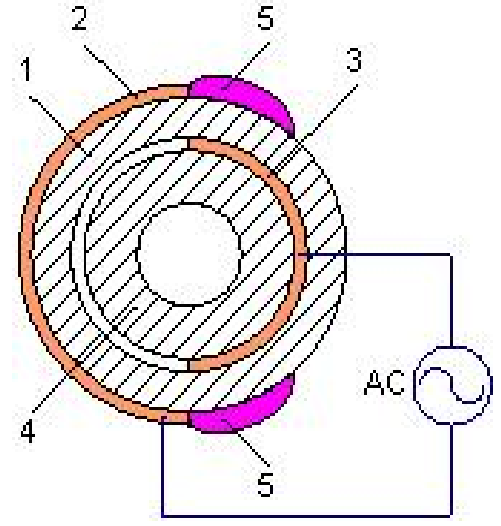

Fig. 4 Schematic of the cylinder model. are connected to a high voltage a.c. source that provides $62 \mathrm{kV}$ peakto-peak positive ramp waveform excitation to the electrodes at a frequency of $1 \mathrm{kHz}$. The high-frequency, highamplitude a.c. voltage is created using a circuit described in [17]. 
The velocity data for the POD analysis was obtained non-intrusively by using PIV measurements. The air upstream of the wind tunnel inlet was seeded with olive oil droplets of nominally 1 micrometer diameter that are produced by a TSI atomizer. A model Y120-15 New Wave Research Nd:Yag laser produced double laser pulses

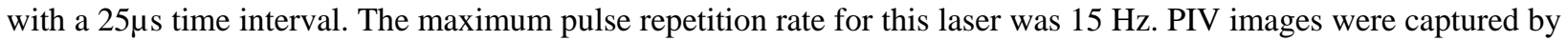
a PIV CAM 10-30 digital camera. TSI Insight 6 software was used to obtain a vector velocity field from each image pair. The interrogation region was approximately 6 cylinder diameters downstream of the end of the cylinder and 6 diameters in the cross-stream direction.

The free stream velocity for the experiments was $U_{\infty}=4 \mathrm{~m} / \mathrm{s}$ which correspond to the Reynolds number of approximately $\mathrm{Re}_{\mathrm{D}}=5,000$. The plasma was turned on and off at the frequency of $4 \mathrm{~Hz}$ (corresponding to a period of $250 \mathrm{~ms}$ ) and a duty cycle of $50 \%$. The whole period of $250 \mathrm{~ms}$ was divided into 50 time steps with $\Delta \tau=5 \mathrm{~ms}$ increment, starting from the moment the plasma actuators were turned on. The PIV data was conditionally sampled at each of these time steps over 200 periods of turning plasma on and off. Thus, for each delay-time step there is an ensemble of 200 acquired velocity fields for the POD analysis.

As was mentioned in the previous section, the TPOD approach should be applied to $u\left(x, y, \tau ; t_{0}\right.$ - fixed) or the phase-locked trajectories (see Figure 2) at the moment the control is turned on or off. When the plasma actuation was turned off, the starting vortices began to develop on both sides of the cylinder. Due to small asymmetry of the cylinder assembly the upper vortex was always slightly stronger than the bottom one, so the flow was naturally locked into the fixed state when the flow control was turned off. The flow control was turned off for $125 \mathrm{msec}$ and due to the periodic nature of the shedding vortical street, by the time the flow control was turned on, it was approximately in the same fixed state as before each actuation-on event. In other words, the slow 4-Hz on/off actuation cycle (compared to the natural shedding frequency of approximately $42 \mathrm{~Hz}$ ) worked as a modulation frequency and effectively had locked the flow into a preferred flow topology at the time the actuation was turned on. Therefore, since the objective of this preliminary work was to collect the data at any fixed flow state and apply the TPOD approach, no special provision was taken to lock the flow into a particular state. Of course, additional experiments with asymmetric actuation are needed to demonstrate extraction of TPOD modes at other fixed states.

\section{Results}

\section{A. TPOD modes}

The experimental 2-D velocity field was processed to extract time-dependent TPOD modes using the snapshot method $^{20}$, as described before. The freestream velocity was subtracted from each velocity field. The first dominant TPOD mode at different time delays from the actuation-on part of the cycle is shown in Figure 5 with the corresponding energy evolution of the first 5 modes shown in Figure 6. It is apparent that the flow undergoes drastic changes when it switches between the natural and controlled states at time delays of 0 and $125 \mathrm{msec}$. Clearly, the flow is phase-locked, so the shedding vortices are clearly seen in Figure 5 at $\tau=160$ and 215, for instance. If the flow was not phase-locked, the POD modes would have been the same regardless of the time delay after the actuation is on or off. The first TPOD mode holds between $60 \%$ and $80 \%$ of the total resolved kinetic energy.

Knowing the TPOD modes at given discrete times, the spatial-only $\Psi(\mathrm{i}, \mathrm{j})$-modes were calculated using (4) and the first six modes for the first TPOD mode, $\varphi_{1}(x, y ; \tau)$, are presented in Figure 7, left plot. The first mode, $\Psi(1,1)$, represents the mean natural flow. The second mode, $\Psi(1,2)$, is a linear combination of the mean flows during the natural and forced states and is related to the "shift" mode [6,21,22]; the third and the fourth modes, $\Psi(1,3)$ and $\Psi(1,4)$, describe the vortex shedding behind the cylinder and higher modes are responsible for the transient regime between the natural and the control cases. Temporal coefficients for these first six $\Psi$-modes, calculated by projecting TPOD mode into the spatial $\Psi$-mode frame of reference, are shown in Figure 7, right plot. These coefficients represent the temporal evolution of the "most-energetic" portion of the flow. Clearly, these modes have hysteresis effects with the forcing being on/off. The vortex shedding modes, $a(1,3)$ and $a(1,4)$, are almost zero during the forcing and experience the Hopf-type bifurcation when the actuation is turned off. Finally, the transient modes, $a(1, \mathrm{j}=5,6)$ are essentially non-zero only during the transient regimes (between control and natural states). 

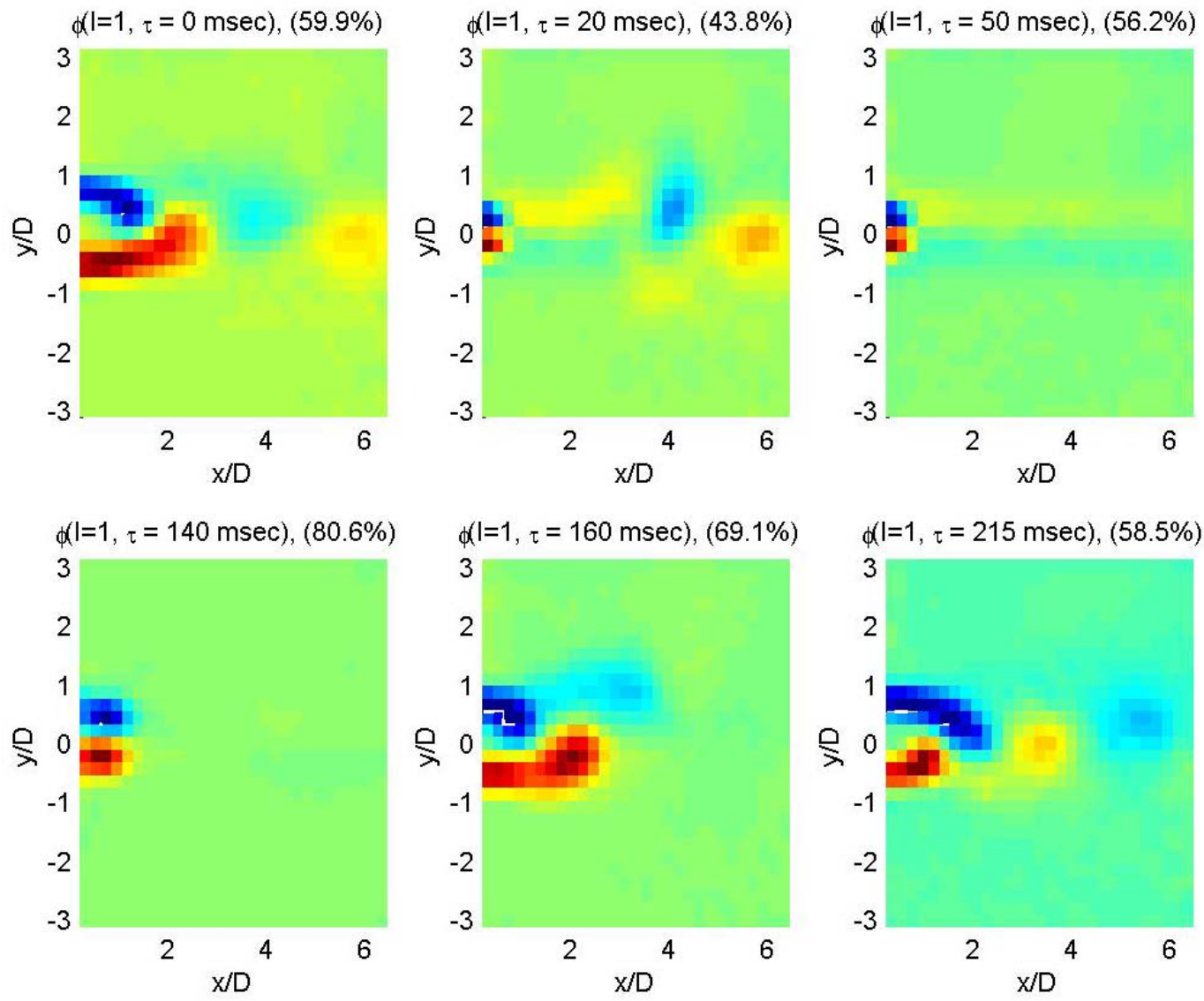

Figure 5. The vorticity of first TPOD mode, $\varphi_{1}(x, y ; \tau)$ at different time delays. The actuation is turned on at $\tau=0 \mathrm{msec}$ and is turned off at $\tau=125 \mathrm{msec}$. Numbers in parenthesis are relative amounts of energy, $\lambda_{1}(\tau) / \sum_{i} \lambda_{i}(\tau)$

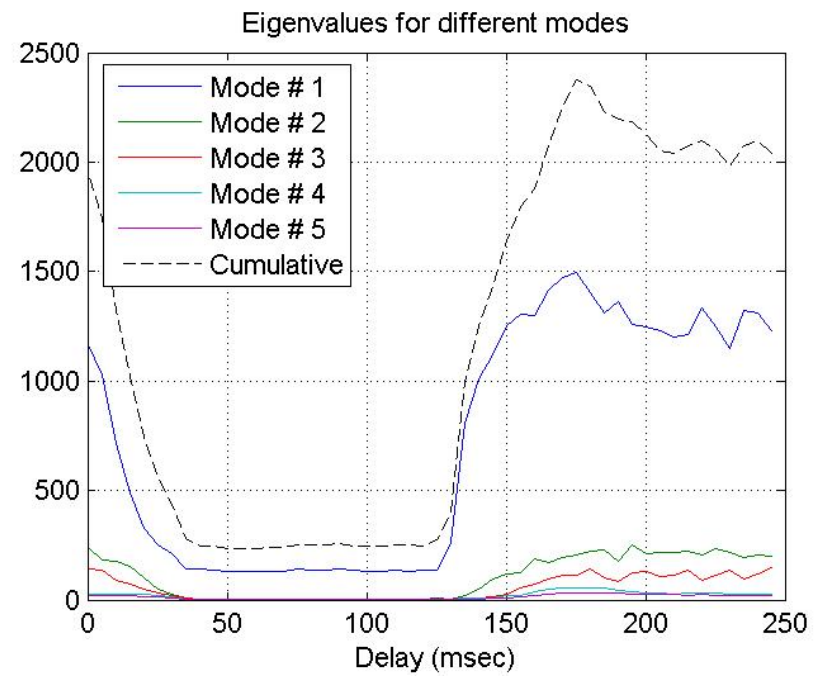

Figure 6. Eigenvalues $\lambda_{i}(\tau)$ and cumulative energies $\sum_{i} \lambda_{i}(\tau)$ at different time delays. 


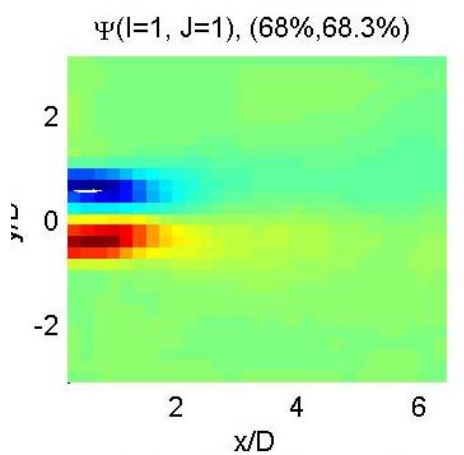

$\Psi(\mid=1, J=3),(7.9 \%, 84.5 \%)$

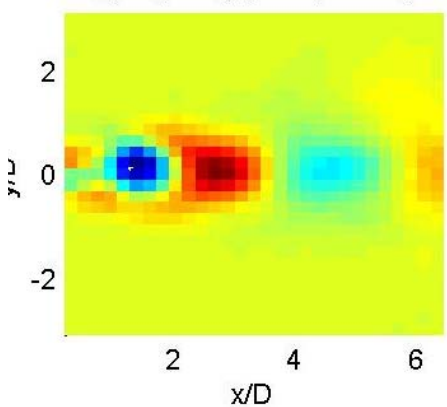

$\Psi(\mid=1, J=5),(3.3 \%, 95.3 \%)$

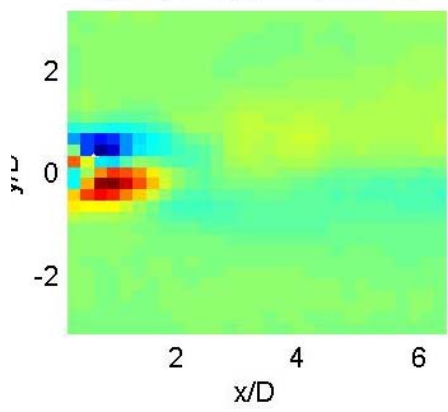

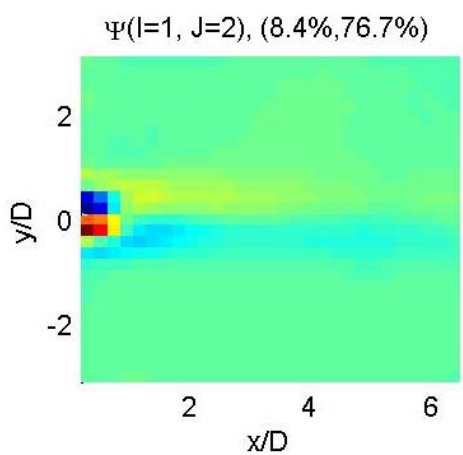

$\Psi(\mathrm{l}=1, \mathrm{~J}=4),(7.5 \%, 92 \%)$

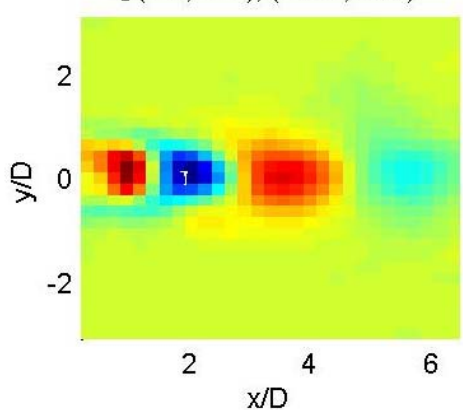

$\Psi(\mathrm{l}=1, \mathrm{~J}=6),(1.6 \%, 96.9 \%)$

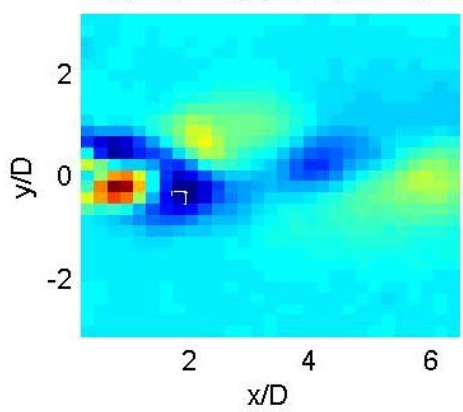

Imode \# 1, Jmode \#1

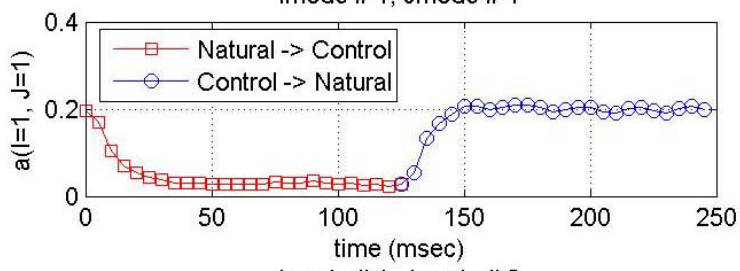

Imode \# 1, Jmode \# 2

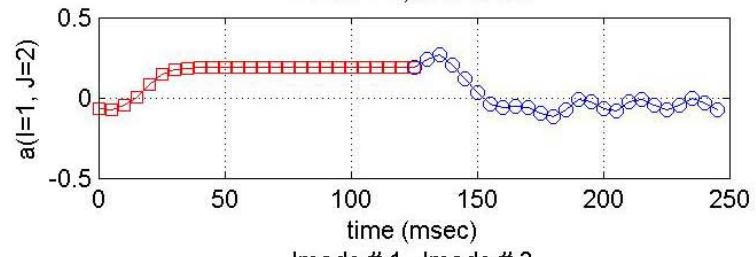

Imode \# 1, Jmode \#3

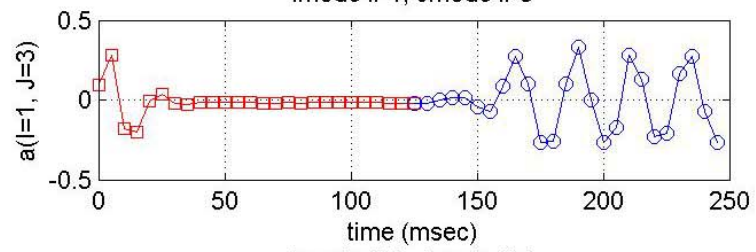

Imode \# 1, Jmode \# 4
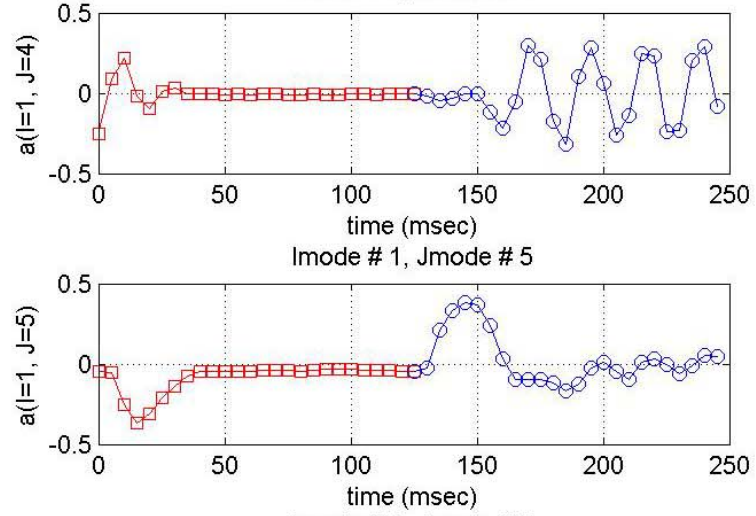

Imode \# 1, Jmode \# 6

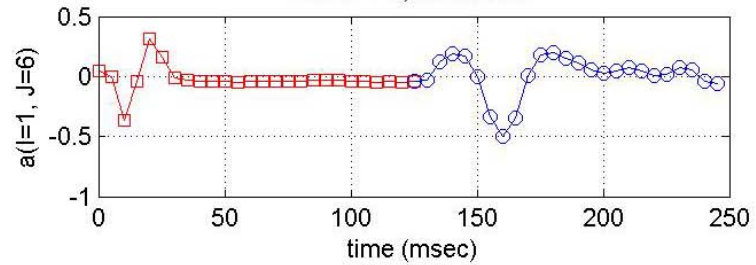

Figure 7. (Left\} Modes $\Psi(\mathbf{i}, \mathbf{j})$ and (right) corresponding coefficients $a(i, j)$, defined in (4).

\section{B. Modeling.}

Experimentally extracted coefficients, $a(i, j)(\tau)$, equation (4), of the first six $\Psi$-modes were used to calculate matrices in (5). To reduce number of unknown coefficients, different combinations of the non-linear terms were tried; the non-linear-term coefficients were calculated using (6) and only those terms with large non-zero coefficients were kept in the right-hand-side of (5). After several attempts, the system (5) was finally written as the following system of ODE's, 


$$
\begin{aligned}
& \frac{d a_{1}(\tau)}{d \tau}=c_{1}+l_{11} a_{1}+l_{12} a_{2}+q_{112} a_{1} a_{2}+q_{122} a_{2}^{2}+b_{1} f \\
& \frac{d a_{2}(\tau)}{d \tau}=c_{2}+l_{21} a_{1}+l_{22} a_{2}+q_{211} a_{1}^{2}+q_{212} a_{1} a_{2}+b_{2} f \\
& \frac{d a_{3}(\tau)}{d \tau}=\left[l_{33}+t_{3}\left(a_{3}^{2}+a_{4}^{2}\right)\right] a_{3}+l_{34} a_{4}+q_{313} a_{1} a_{3}+b_{3} f \\
& \frac{d a_{4}(\tau)}{d \tau}=l_{43} a_{3}+\left[l_{44}+t_{4}\left(a_{3}^{2}+a_{4}^{2}\right)\right] a_{4}+q_{414} a_{1} a_{4}+b_{4} f \\
& \frac{d a_{5}(\tau)}{d \tau}=\left[l_{55}+t_{5}\left(a_{5}^{2}+a_{6}^{2}\right)\right] a_{5}+l_{56} a_{6}+q_{515} a_{1} a_{5}+t_{5511} a_{5} a_{1}^{2}++t_{5512} a_{5} a_{1} a_{2}+b_{5} f \\
& \frac{d a_{6}(\tau)}{d \tau}=l_{56} a_{5}+\left[l_{66}+t_{5}\left(a_{5}^{2}+a_{6}^{2}\right)\right] a_{5}+q_{616} a_{1} a_{6}+t_{6611} a_{6} a_{1}^{2}++t_{6612} a_{6} a_{1} a_{2}+b_{6} f
\end{aligned}
$$

To model the temporal evolution of the first two modes responsible for the mean flow evolution in the presence of the forcing, only the linear and quadratic terms were explicitly included. Equations for the vortex shedding modes, $a_{3}$ and $a_{4}$, have simple stabilizing cubic terms, $t_{3}\left(a_{3}^{2}+a_{4}^{2}\right) a_{3}$ and $t_{4}\left(a_{3}^{2}+a_{4}^{2}\right) a_{4}$; an interaction with the mean flow, $a_{1}$, was modeled by including only quadratic terms $q_{313} a_{1} a_{3}$ and $q_{414} a_{1} a_{4}$. Equations for the transient terms, $a_{5}$ and $a_{6}$, have similar stabilizing cubic terms, $t_{5}\left(a_{5}^{2}+a_{6}^{2}\right) a_{5}$ and $t_{6}\left(a_{5}^{2}+a_{6}^{2}\right) a_{6}$ and interaction with the mean-flow modes, $a_{1}$ and $a_{2}$, was modeled as a cubic interaction only. The forcing coefficient, $f$, was set to 0 for the no-forcing case and 1 for the forcing case. Temporal derivatives at discrete times, $\mathrm{d} a_{\mathrm{i}}(\tau) / \mathrm{d} \tau$, were numerically calculated using the second-order finite difference, $\frac{d a_{i}(\tau)}{d \tau} \approx \frac{a_{i}(\tau+\Delta \tau)-a_{i}(\tau-\Delta \tau)}{2 \Delta \tau}$, with $\Delta \tau=5$ msec, and the least-squared procedure was used to find the best fit for all unknown coefficients. The resulted numerical values are given in Table 1.

The system (7) was then solved numerically with these values of coefficients in the time interval [0..250 msec] with a numerical time step of $0.1 \mathrm{msec}$. The forcing term was set $f=1$ between 0 and 125 msec and $f=1$ between 125 and 250 msec. Results of the modeling and the comparison with experimentally obtained coefficients are presented in Figure 8. The system of equations (7) correctly predicts most of the essential features of the temporal evolution of the first six modes with the forcing on/off. The natural vortex shedding frequency was calculated to be $l_{43} /(2 \pi)=44.6 \mathrm{~Hz}$, which is close to the experimentally observed frequency of approximately $42 \mathrm{~Hz}$. The first and second modes, $a_{1}(\tau)$ and $a_{2}(\tau)$, were correctly predicting the mean-flow changes with the forcing turned on and off, but exhibited a moderate decaying-oscillation behavior when the forcing was switched off. The modeled values of the vortex-shedding modes, $a_{3}(\tau)$ and $a_{4}(\tau)$, were in general correctly predicting the experimental values, but were slightly out-of-phase compared to the experimentally-measured values. The transient terms, $a_{5}(\tau)$ and $a_{6}(\tau)$, while predicting correct trends, miss some details of high-oscillation features when the control was turned off.

Table 1. Coefficients in system (7)

\begin{tabular}{|l|l|l|l|}
\hline \multicolumn{1}{|c|}{ Linear terms } & \multicolumn{1}{c|}{ Quadratic terms } & \multicolumn{1}{c|}{ Cubic terms } & Forcing \\
\hline$c_{1}=-5, c_{2}=32.7$, & $q_{112}=-45.4, q_{122}=47.2$, & & $b_{1}=-4.2$ \\
$l_{11}=30 ; l_{12}=36.5$, & $q_{211}=-361.1, q_{212}=304$ & & $b_{2}=-7.3$ \\
$l_{21}=-111.7 ; l_{22}=-120.5$ & & & \\
$l_{33}=-340, l_{34}=-280$, & $q_{313}=3020 ; q_{414}=-367$ & $t_{3}=-3400, t_{4}=-350$ & $b_{3}=2.0$ \\
$l_{34}=280, l_{44}=101.2$ & & & $b_{4}=2.0$ \\
$l_{55}=-246, l_{56}=160$, & $q_{515}=6093, q_{616}=7446$ & $t_{5}=-400, t_{6}=-400$ & $b_{5}=-1.9$ \\
$l_{65}=-160, l_{66}=-510$ & & $t_{5511}=-23707$, & $b_{6}=-20.9$ \\
& & $t_{6512}=-886$ & \\
& & $t_{6612}=6697$ & \\
\hline
\end{tabular}



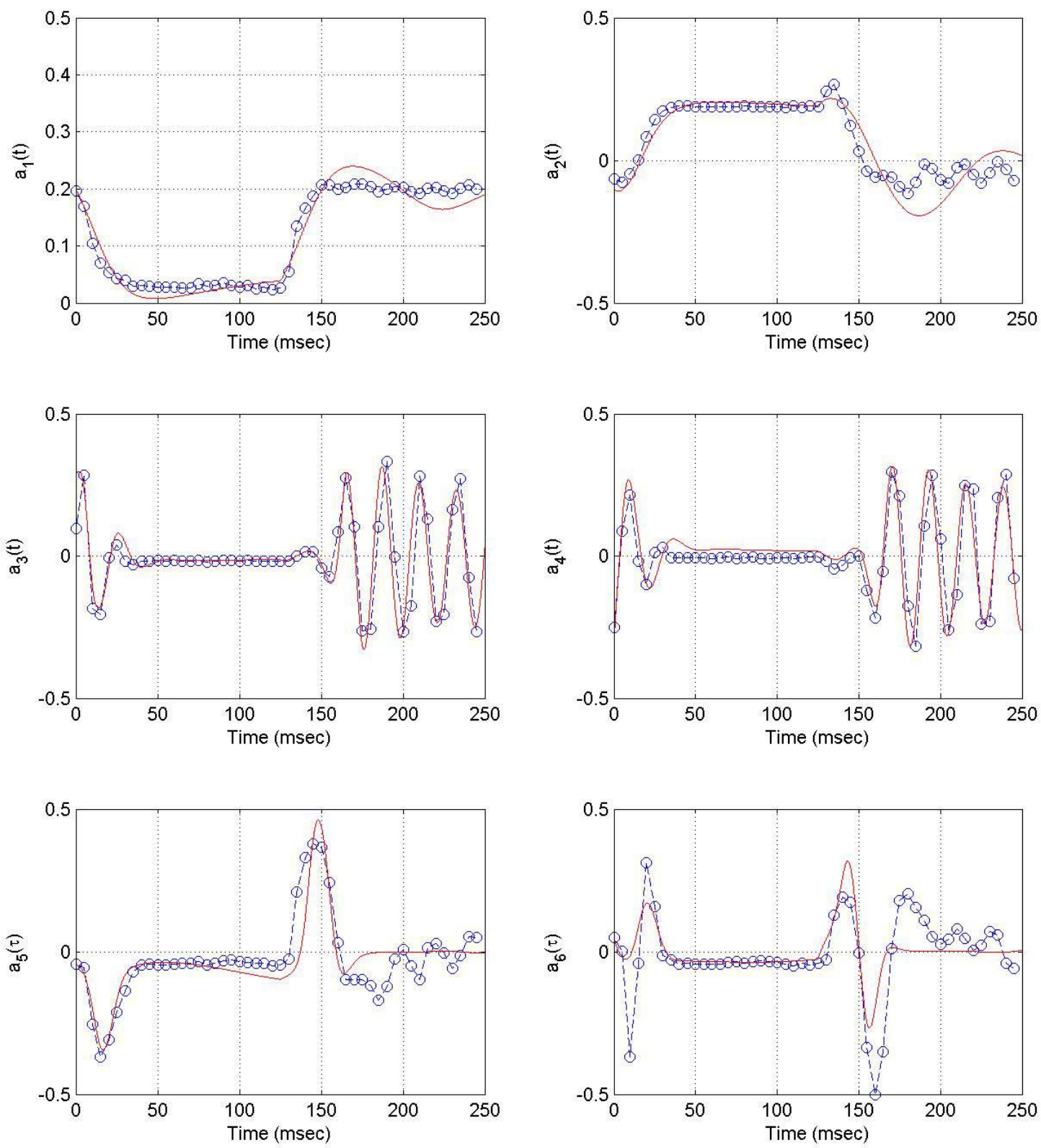

Figure 8. Numerical solution of the system (7) (solid red lines) and experimental results (blue dashed lines with circles). Forcing is turned on at $0 \mathrm{msec}$ and turned off at $125 \mathrm{msec}$.

The main factor contributing to the existing small differences between the predicted and measured values of the temporal coefficients is most likely the numerical calculation of the temporal derivatives, $\mathrm{d} a_{\mathrm{i}}(\tau) / \mathrm{d} \tau$, since the coefficients are given at discrete times. Also, adding more non-linear terms in (7) will probably improve the model, but obviously will make it more complicated and subject to artificial numerical instabilities.

\section{Discussion and future work.}

This paper has demonstrated a novel technique, Temporal POD (TPOD), to extract the optimum set of eigenmodes and the temporal evolution of these modes from the experimental data with the presence of the flow control. Retaining the time in TPOD modes allows one to extract important information about the system trajectories in the optimum frame of reference. The technique was applied to the wake flow downstream of a cylinder at moderate Reynolds number with active flow control in the form of two plasma actuators. The extracted temporal 
coefficients were used to develop a non-linear model with various linear, quadratic and cubic terms; the least-square analysis was used to analyze the importance of individual terms. The developed model showed excellent promise in capturing the dynamics of the natural, controlled and transient states of the flow, as well as hysteresis effects with the presence of the flow control. It is important to note that in order to build the model, no additional knowledge about the flow dynamics was used.

While we were encouraged by these promising results, this work is obviously only the first step toward the larger goal of achieving a complete model of the flow with flow control. Additional studies will include experiments with different forcing levels to verify the range of applications of the model and/or to update the model at different forcing regimes. Also, to extract TPOD modes for other fixed states, additional measurements with asymmetric plasma excitation should be taken. Ultimately, the model with the closed-loop control will be tested and results will be compared with experiments.

\section{Acknowledgements}

The authors would like to thank Alexey Kozlov for conducting plasma experiments and PIV measurements for this paper.

\section{References}

${ }^{1}$ Berkooz, G., Holmes, P., \& Lumley, J. L. 1993, "The proper orthogonal decomposition in the analysis of turbulent flows,” Ann. Rev. Fluid Mech., 25, 539-575.

${ }^{2}$ Holmes, P., Lumley, J. L., \& Berkooz, G., 1996, Turbulence, Coherent Structures, Dynamical Systems and Symmetry, Cambridge University Press.

${ }^{3}$ Lumley, J., 1970, Stochastic Tools in Turbulence, Academic, New York.

${ }^{4}$ Aubry, N., Holmes, P., Lumley, J. L., \& Stone, E., 1988, “The dynamics of coherent structures in the wall region of the turbulent shear layer,” J. Fluid Mech. 192, 115-175.

${ }^{5}$ Ukeiley, L. S., Cordier, L., Manceau, R., Delville, J., Glauser, M. N., \& Bonnet, J.P., 2001, "Examination of largescale structures in a turbulent plane mixing layer. Part 2. Dynamical systems model,” J. Fluid Mech., 441, 67108.

${ }^{6}$ Siegel, S. G., Seidel, J., Fagley, C., Luchtenburg, D. M. \& McLaughlin, T., 2008, “Low-dimensional modeling of a transient cylinder wake using double proper orthogonal decomposition,” J. Fluid Mech., 610, 1-42.

${ }^{7}$ S. Siegel, K. Cohen ., J. Seidel, S Aradag, and T. McLaughlin, “Low Dimensional Model Development using Double Proper Orthogonal Decomposition and System Identification”, 4th Flow Control Conference, June, 2008, AIAA-2008-4193.

${ }^{8}$ R. C. Camphouse, J. H. Myatt, R. F. Schmit, M. N. Glauser, J. M. Ausseur, M.Y. Andino, and R. D. Wallace, “A Snapshot Decomposition Method for Reduced Order Modeling and Boundary Feedback Control”, Paper AIAA2008-4195, 4th Flow Control Conference, Seattle, Washington, 2008.

${ }^{9}$ Rowley, C. W., 2005, “Model reduction for fluids using balanced proper orthogonal decomposition”, Int. J. Bifurcation Chaos 15 (3), 997-1013.

${ }^{10}$ Juang, J.N., Pappa, R.S." An eigensystem realization algorithm for modal parameter identication and model reduction,” J. Guid. Contr. Dyn. 8(5), 620-627, 1985.

${ }^{11}$ Z. Ma, S. Ahuja, and C. W. Rowley ,’Reduced order models for control of fluids using the Eigensystem Realization Algorithm”, Theoret. Comput. Fluid Dyn. (submitted), June 2009.

${ }^{12}$ Williamson, C.H.K, "Vortex dynamics in the cylinder wake,” Annu. Rev. Fluid Mech., 28, pp. 477-539.

${ }^{13}$ D. M. Luchtenburg, B. Gunther, B. R. Noack, R. King and G. Tadmor,2009, ”A Generalized Mean-Field Model of The Natural and High-Frequency Actuated Flow Around a High-Lift Configuration”, J. Fluid Mech., Vol. 623, Pp. 283-316.

${ }^{14}$ G. Tadmor, B.R. Noack, M. Morzynski and S. Siegel, "Low-Dimensional Models For Flow Control. Part II. Control and Dynamic Estimation”, $2^{\text {nd }}$ Flow Control Conference, June, 2004, AIAA-2004-2409.

${ }^{15}$ J.M. Aussier and J.T. Pinier, “Toward Closed-Loop Feedback Control of the Flow over NACA-4412 Airfoil”, $43^{\text {rd }}$ AIAA Aerospace Sciences Meeting and Exhibit, Jan 2005, AIAA- 2005-343.

${ }^{16}$ Samimy, M., Debiasi, M., Caraballo, E., Serrani, A., Yuan, X., Little, J. and Myatt, J., 2007, "Feedback control of subsonic cavity flows using reduced-order models”, J. Fluid Mech. 579, 315-346.

${ }^{17}$ Thomas, F., Corke, T. C., Iqbal, M., Kozlov, A. and Schatzman, D., "Optimization of SDBD Plasma Actuators for Active Aerodynamic Flow Control” AIAA Journal, Vol. 47, No. 9, 2009, pp. 2169-2178.

${ }^{18}$ Thomas, F. O. Kozlov, A. and Corke, T. C., 2008, "Plasma Actuators for Cylinder Flow Control and Noise Reduction,” AIAA Journal, 46(8), pp.1921-1931. 
${ }^{19}$ Kozlov, A. and Thomas, F. O., 2009, “Active Noise Control of Bluff Body Flows Using Dielectric Barrier Discharge Plasma Actuators,” AIAA paper 2009-3245, 15 ${ }^{\text {th }}$ AIAA/CEAS Aeroacoustics Conference, Miami, FL.

${ }^{20}$ Sirovich ,L, 'Turbulence and the Dynamics of Coherent Structure: I, II and III', Quarterly Applied Mathematics, (1987), 45, p. 561.

${ }^{21}$ Gerhard, J., Pastoor, M., King, R., Noack, B. R., Dillmann, A., Morzynski, M. and Tadmor, G., “Model based control of vortex shedding using low-dimensional galerkin models”, AIAA CP 2003-4261.

${ }^{22}$ Siegel, S., Cohen, K. and McLaughlin, T.,’Feedback control of a circular cylinder wake in experiment and simulation”, AIAA Paper 2003-3569. 